\title{
GHRELIN EXPRESSION IN MAST CELLS OF INFANT LUNG WITH RESPIRATORY DISTRESS SYNDROME
}

\author{
K. Ivanova', I. Stefanov', I. Ivanova², J. Ananiev', M. Gulubova' \\ 'Department of General and Clinical Pathology, Medical Faculty, Trakia University - Stara Zagora, Bulgaria \\ ${ }^{2}$ Department of Anatomy, Medical Faculty, Trakia University - Stara Zagora, Bulgaria
}

\begin{abstract}
This article sheds light on some features of ghrelin (GHR)- and tryptase (Try)positive mast cells (MCs) distribution in human lung of preterm newborns with respiratory distress syndrome (RDS). GHR possessed anti-inflammatory activity and reliable therapeutic properties in some lung diseases. So far, GHR expression has been defined predominantly in neuroendocrine cells of bronchial mucosa in fetal and infant lungs. Lung tissue from 8 dead newborns with RDS were investigated immunohistochemically with antiGHR and anti-Try antibodies. The number of GHR+ and Try+ MCs was determined in three locations -bronchi, bronchiole and in alveolar septa. MCs were more numerous around main bronchi with diminishing numbers around bronchiole and in alveolar septa. The number of MCs in the latter was increased in newborns with pneumonia. The number of GHR+ MCs in alveolar septa was lower in newborns with RDS as compared to newborns with $R D S$ combined with pneumonia $(2.83 \pm 1.13$ vs $4.81 \pm 2.6, p<0.001)$. The amount of Try+ MCs along bronchial wall was significantly more than GHR+ MCs in RDS newborns (6.97 \pm 4.53 vs $3.85 \pm 4.30, p=0.001$ ). It could be supposed that pulmonary MCs increased in newborn lungs in inflammatory process. MCs in human lung contained GHR peptide that had immunomodulatory function and participated in hormone regulation of inflammation.
\end{abstract}

Key words: ghrelin, mast cells, respiratory distress syndrome

Corresponding author: Koni Ivanova, MD, PhD, Department of General and Clinical Pathology, Medical Faculty, Trakia University, 11 Armeiska Str., Bg - 6000 Stara Zagora, phone: 0035942664421, e-mail: koni_ivanova@yahoo.com

REVISED: 03 November 2019, ACCEPTED: 15 November 2019

\section{INTRODUCTION}

espiratory distress syndrome (RDS) known also as hyaline membrane disease (HMD) is an acute pulmonary disease that causes mortality and morbidity in preterm newborns [1]. The basic defect causing the RDS in preterm babies is represented by immaturity of the lungs, particulary of type II pneumocytes where pulmonary surfactant is synthesized. According to Grappone and Messina (2014) surfactant deficit and immature lung structure with reduced alveolization and excess connective tissue matrix lead to alveolar instability, collapse, atelectasis, acidosis, hypoxia, capillary leak edema and alveoli necrosis; hyaline membranes formation occurs within the wall of the bronchial terminals and the alveolar ducts [1]. In RDS influx of protein rich fluid into the air spaces occurs as a result of increased permeability of the alveolar-capillary barrier. According to Bitterman, injured alveolar epithelium in this disease is followed by insufficient epithelial repair which may cause fibrosis [2]. The collapse of alveoli, 
known as atelectasis, allows for exchange of gases through the walls of terminal bronchioles, structures that are not suitable for that purpose. The formation of hyaline membranes reduces the passage of oxygen from the alveolar spaces across the alveolar wall into the pulmonary vasculature. In addition, extravasation of blood into the respiratory airways, combined with the collapse of the alveoli, further contributes to the consolidation of the newborn lungs. Many other factors are known and are important for increasing the risk for developing RDS such as gender of newborns (males : females - 2:1), previous birth of newborns with HMD, perinatal asphyxia, etc [3]. Because the risk of RDS depends on the race, the ethnic group, and is increased in mono- or hetero zygotic twins, genetic factors have been suggested [4].

Ghrelin is a 28-amino acid orexigenic peptide described as a strong physiologic growth hormone $(\mathrm{GH})$ secretagogue which interacts with the growth hormone secretagogue receptor 1a (GHSR1a) [5]. In adults, ghrelin production was first established in gastric X/A-like cells, then in other organs including pituitary, hypothalamus, kidney, heart, placenta and immune cells [6]. This hormone is involved in many physiological processes, including control of gastric motility and acid secretion in animals and humans. It participates also in stimulation of appetite, increasing the food intake, decreasing the energy expenditure and lipogenesis. Its discovery has contributed to the elucidation of a new mechanism of the regulation of growth hormone $(\mathrm{GH})$ secretion. Hattori et al., reported that GHR and its receptor (GHR-R) were also expressed in human immune cells such as $\mathrm{T}$ lymphocytes, B lymphocytes and neutrophils, monocytes and in lymph nodes [7]. GHR leads to inhibition of the expression of proinflammatory cytokines such as IL-1b, IL-6 and TNF-a in T lymphocytes and monocytes in humans. Antiapoptotic effect of ghrelin also has been discovered. This effect of GHR is achieved by inhibiting TNF-a induced apoptosis and suppressing caspase-3 activation in osteoblastic cell lines. The anti-apoptotic effect of GHR has also been revealed in other cells such as pancreatic b-cells, enterocytes, adipocytes, cardiomyocytes and endothelial cells and where it is mediated via phosphatidyl inositol 3-kinase (PI3K) activation and glycogen synthase kinase-3b (GSK3b) suppression, and stabilization of beta-catenin. It was suggested that GHR has an anti-apoptotic effect on immune cells, although such evidence has not been published yet [7].

Ghrelin immunoreactivity in MCs was established in the serosal, muscular, submucosal and mucosal layers of the rat stomach [8]. Thus, the authors suggested that these cells are probably an important source of GHR with a variety of physiological functions, such as regulation of stomach epithelial, glandular and muscular functions.

The important role of MCs not only in allergic diseases but also in a number of non-allergic immune reactions has been widely studied. The main reason for that is the variety of MCs mediators involved in the pathogenesis of different diseases including lung diseases characterized by allergic, acute and chronic inflammatory, fibrotic and neoplastic processes [9-13]. These mediators are usually subdivided into preformed and newly generated after activation. Preformed mediators are histamine, heparin, chondroitin sulfate E, serotonin, tryptase, chymase, carboxypeptidase, cytokines such as IL-4 and IL-15, and growth factors (basic fibroblast growth factor, bFGF, tumor necrosis factor alpha (TNF- $\alpha$ ), nerve growth factor (NGF) [1418]. The newly synthesized mediators released after activation are prostaglandin D2 and leukotrienes, inflammatory cytokines and chemokines [19, 20]. Mast cell tryptase is a neutral serine protease, acting by protease activated receptors (PAR) belonging to the G-protein-coupled receptors [21]. Berger et al. established PARs localization in airway epithelial and smooth muscle cells, terminal bronchial epithelium, type II pneumocytes, endothelial and vascular smooth muscle cells, and as well as in MCs within the respiratory tract [22].

According to Smith et al. immunohistochemical detection of tryptase within human lung mast cells is more reliable than toluidinblue staining [23]. In normal lungs of infants, the authors observed the more number of MCT in the outer layer than in the epithelial layer of the airways [23].

MCs involvement in pathophysiological mechanism of pneumonia was investigated mainly in rodents, which are suitable experimental model. For example, Mycoplasma pulmonis causes natural murine respiratory disease with manifestations similar to those in humans with Mycoplasma pulmonis infection [24]. Many authors reported that Chlamydia pneumoniae induces asimilar lung pathology in humans and rodents. In this regard, it was found out that in the airway, Chlamydia pneumoniae first infects alveolar macrophages and airway epithelial cells, leading to secretion of proinflammatory cytokines and chemokines that result in an influx of inflammatory cells, such as monocytes, macrophages, neutrophils Tand B lymphocytes [25-27].

The lack of information about the mast cell participation and the role of GHR in development of RDS motivated us to undertake this study. 


\section{MATERIALS AND METHODS}

\section{Materials}

Newborns' data were collected and assessed from the archives of the Department of General and Clinical Pathology, Medical Faculty, Trakia University, Stara Zagora in the period from 2016-2017. Lung tissue from 8 dead newborns (from 2 days to 44 days old) with RDS where investigated immunohistochemically with anti-GHR and anti-Try antibodies.

\section{Immunohistochemistry}

Biopsy specimens were treated for routine histology (staining with hematoxylin and eosin) and for light microscopcopy immunohistochemistry. For immunohistochemical staining, the paraffin blocks were prepared using tumor tissues from the periphery of the tumor adjacent to the normal tissues. Paraffin sections $5 \mu \mathrm{m}$ thick were dewaxed in two xyllenes (for $30 \mathrm{~min}$ each at $56^{\circ}$ ) and were rehydrated in ethanol. Then the sections were soaked in $10 \%$ sucrose in distilled water, overnight. Later, they were washed in $0.1 \mathrm{M}$ phosphate buffered saline (PBS), $\mathrm{pH} 7.4$, incubated in $1.2 \%$ hydrogen peroxide in methanol for $30 \mathrm{~min}$, and rinsed in $0.1 \mathrm{M} \mathrm{PBS}, \mathrm{pH}$ 7.4 , for $15 \mathrm{~min}$. Then the slides were incubated in a humid chamber until night, at room temperature with antibody monoclonal mouse anti-human mast cell Tryptase clone: AA1, M 7052 (DAKO, Denmark) and rabbit anti-human antibody against polyclonal ghrelin sc-50297 (Santa Cruz, Biotechnology, USA). After washing three times in PBS, the slides were incubated with En-VisionTM detection system (DAKO) for $60 \mathrm{~min}$, then visualized with diaminobenzidine and counterstained with Mayer's hematoxylin.

\section{Cell counting}

The number of GHR+ and Try+MCs were counted in three locations - around bronchi, around bronchiole and in alveolar septa, on five fields of vision at a magnification ( $\times 320,0.74 \mathrm{~mm}^{2}$ area).

\section{RESULTS}

\section{Hyaline membrane morphology}

In lung sections (H\&E staining) hyaline membrane formations were observed in alveoli, alveolar ducts or respiratory bronchioles (resulted from fibrin, cellular debris, red blood cells). Hyaline membranes appear like an eosinophilic, amorphous material, lining or filling the alveolar spaces and blocking the gases exchange.

\section{Immunohistochemical features}

The immunohistochemical study was performed to identify GHR+ MCs by using anti-tryptase antibody to confirm that observed GHR+ cells are MCs. The immunohistochemical detection of Try and GHR expression showed that the ratio of $\mathrm{MC} \mathrm{GHR+} \mathrm{to}$ $\mathrm{MC}+$ was similar in both lungs with RDS only and with RDS plus pneumonia (Table 1). In the alveolar septa and bronchiolar wall, the number of MCs GHR+ was significantly higher than MCsTry+, whereas in bronchi, MCsTry+ were more than GHR+cells. These results showed that all MCs in the alveolar septa and bronchiolar wall express GHR, whereas in the bronchial wall, only half of the MCsTry+ expresses GHR.

When lungs with RDS only were compared to the lungs with RDS plus pneumonia, the immunohistochemical expression of Try and GHR showed that in the alveolar septa of lungs with RDS only, the number of MCsGHR+ was lower than in patients who suffered from both RDS and pneumonia $(2.83 \pm 1.13 \mathrm{vs}$. $4.81 \pm 2.6, p<0.001)$. The amount of Try+ MCs along bronchial wall was significantly more than $\mathrm{GHR}+$ MCs in RDS newborns $(6.97 \pm 4.53$ vs. $3.85 \pm 4.30$, $p=0.001)$. We also estimated that in patients with RDS plus pneumonia, the amount of MCsGHR+ and MCsTry+ in bronchioles was higher than in RDS only but without statistical significance. Cells distributions are shown on Figure 1 and Figure 2.

Table 1. Mast cell density (number/mm²) in different lung compartments in patients who suffered from both RDS and RDS + Pneumonia

\begin{tabular}{|l|c|c|c|c|c|c|}
\hline $\begin{array}{l}\text { Mast cell num- } \\
\text { ber/mm }\end{array}$ & RDS alveoli & bronchioles & bronchi & $\begin{array}{c}\text { RDS+Pneu } \\
\text { alveoli }\end{array}$ & bronchioli & bronchi \\
\hline MCsGHR+ & $2.83 \pm 1.13$ & \multicolumn{2}{|c|}{$p<0.001$} & \multirow{2}{*}{$4.81 \pm 2.60$} & $3.39 \pm 2.48$ & $3.35 \pm 4.06$ \\
\cline { 3 - 6 } & & $2.26 \pm 2.03$ & $3.85 \pm 4.30$ & & \\
\hline \multirow{2}{*}{ MCsTry+ } & $0.335 \pm 0.22$ & $0.617 \pm 0.33$ & $p=0.53$ & $1.58 \pm 0.57$ & $1.35 \pm 1.02$ & $7.59 \pm 6.60$ \\
\hline
\end{tabular}




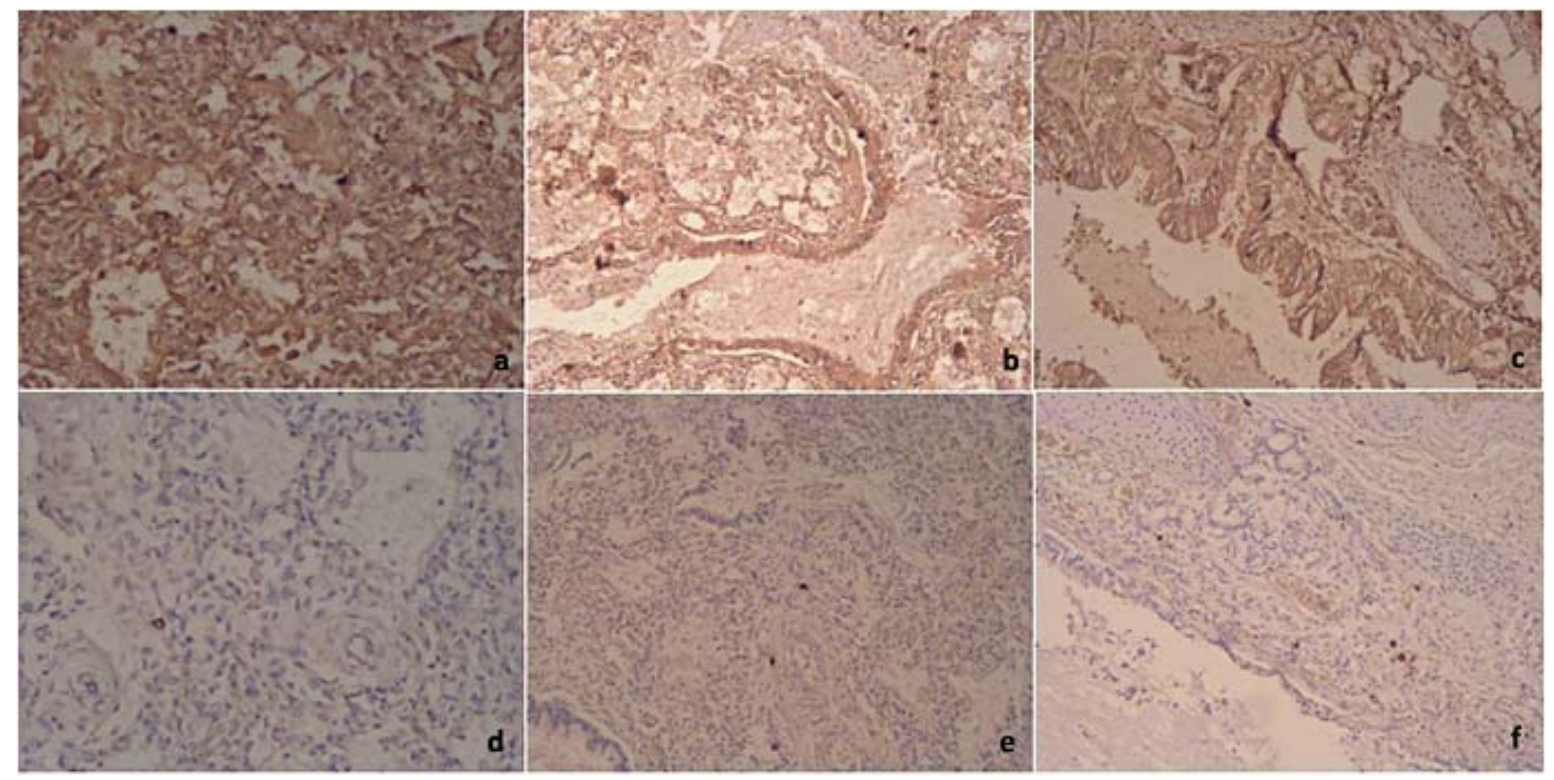

Fig. 1. a) Ghrelin-positive mast cells in alveoli of case with RDS (x 200);b) Ghrelin-positive mast cells in bronchioles of case with RDS (x 200 ); c) Ghrelin-positive mast cells in bronchi of case with RDS (x 200); d) Tryptase-positive mast cells in alveoli of case with RDS (x 200); e) Tryptase-positive mast cells in bronchioles of case with RDS (x 100); f) Tryptase-positive mast cells in bronchi of case with RDS (x 100)

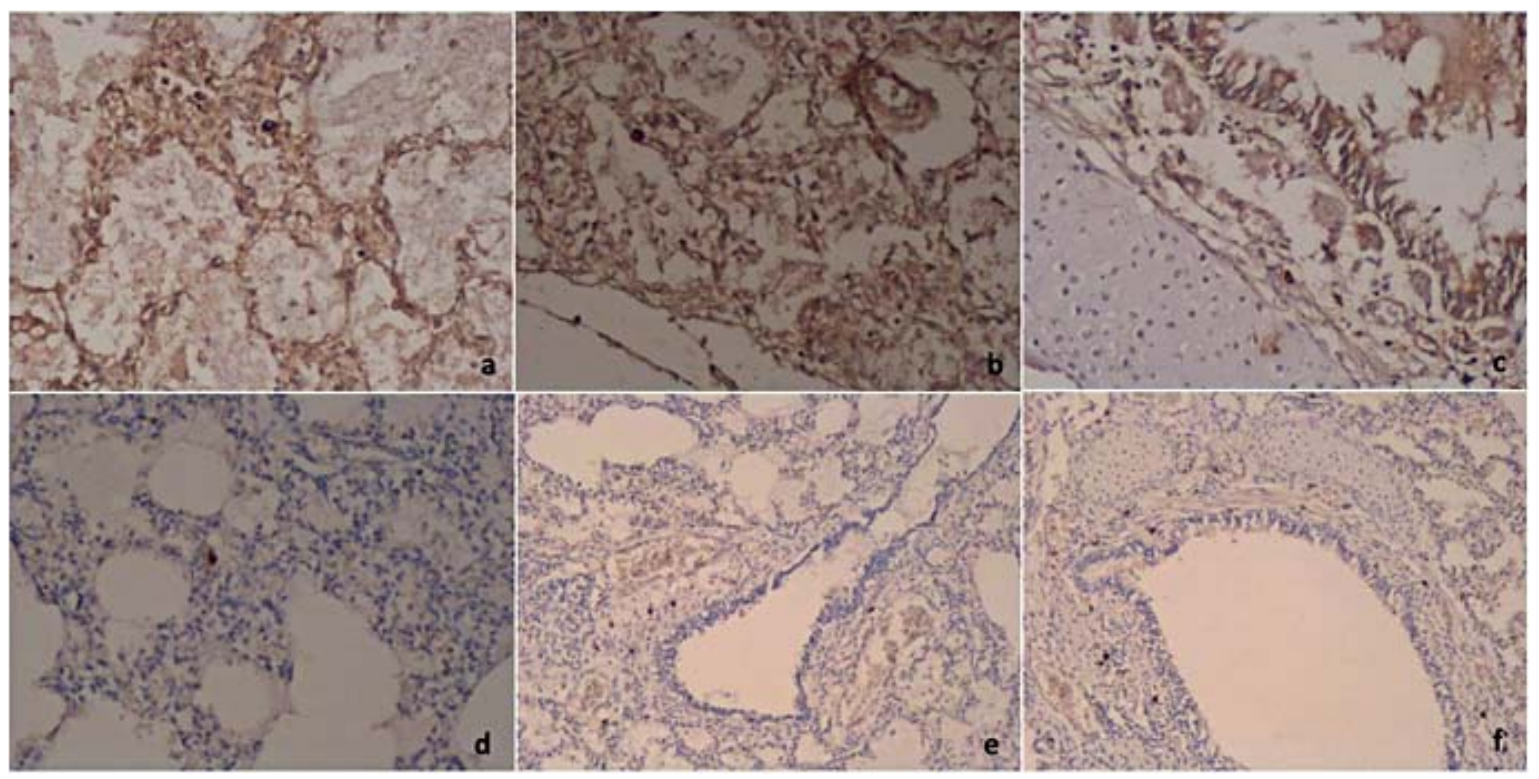

Fig. 2. a) Ghrelin-positive mast cells in alveoli of case with RDS and pneumonia (x 200); b) Ghrelin-positive mast cells in bronchioles of case with RDS and pneumonia (x 200); c) Ghrelin-positive mast cells in bronchi of case with RDS and pneumonia (x 200); d) Tryptasepositive mast cells in alveoli of case with RDS and pneumonia (x 200); e) Tryptase-positive mast cells in bronchioles of case with RDS and pneumonia (x 100); f) Tryptase-positive mast cells in bronchi of case with RDS and pneumonia (x 100)

\section{DISCUSSION}

The MCs number deserves attention in order to clarify the biological and pathological role of these cells. For example, homeostatic regulation of lung vasculature, alveolar defense, modulation of immune response and involvement of mast cells in lung diseases are some of the potential functions that MCs may exert.

So far, the number of metachromatic cells was estimated according to their localization in adult human lung of pa- tients with idiopathic bronchiolitis obliterans organizing pneumonia (BOOP) and in control patients [28]. The lung tissues from patients with BOOP contained higher numbers of metachromatic mast cells ranging from 66.02 to 162.25 (median of 126.9) cells per square millimeter of tissue section, significantly higher than control subjects. It was reported that $73 \%$ of the mast cells were detected in the alveolar septa, $18 \%$ within alveoli and $4 \%$ among alveolar lining cells. The tissue from control patients contained lower numbers of metachromatic mast cells with a range of 12.69 to 41.88 (31.7). Ninety-seven percent 
(97\%) of these cells were observed in alveolar septa and $3 \%$ in vascular walls, but no metachromatic cells were found within the alveoli. In the current study we used anti-tryptase antibody to identify mast cells which express GHR as we did in our previous study in rat stomach [8]. It is known that Try plays a key role in allergic diseases by amplifying the responses of mast cells to different stimuli including allergens [29]. In asthma, the release of MCmediators such as histamine, prostaglandin D2 and leukotriene $\mathrm{C} 4$, induces typical asthmatic symptoms such as bronchoconstriction, mucus secretion and mucosal edema [30]. The role of Try in RDS has not been established yet.

In regards to the GHR expression, it was observed in neuroendocrine cells only of the fetal and infant lung and occasionally in adult lung [31]. The mentioned authors detected that in the postnatal period, during the first 2 years of life, single GHR positive endocrine cells were observed in the bronchial mucosa (mean - 4.0 ghrelin-positive cells per bronchial section). However, the authors did not identify other GHR immunopositve cells in the bronchial wall or in the terminal parts of the bronchial tree [31].

In the current study, we revealed that in the alveolar septa and bronchiolar wall, the number of MCsGHR+ was significantly higher than MCsTry+, whereas in bronchi, MCsTry+ were more than GHR+cells. The results showed that all MCs in the alveolar septa and bronchiolar wall express GHR and allowed us to suggest that these cells have a metabolic pathway to synthesize the hormone. On the other hand, the higher MCsGHR+ number than MCsTry+ suggests that other cells different from MCs also may express GHR in lung and they remain to be defined in further investigations. These results support the study by Hattori et al., where was reported that GHR and its receptors were expressed in human immune cells such as monocytes, T lymphocytes, B lymphocytes and neutrophils in lymph nodes. It was found out that GHR inhibit the expression of proinflammatory cytokines such as IL-1b, IL-6 and TNF-a in T lymphocytes and monocytes in humans [7].

The interesting finding in the current study is that not all but only half of the Try positive mast cells expressed GHR in the bronchial wall. Therefore, in bronchial wall, MCsTry+ are significantly more than MCsGHR+, whereas in alveolar and bronchiolar wall all MCs are GHR positive. This finding allows us to suggest the potential influence of GHR on hyaline membrane deposition and collapse of alveoli, not adequately coated with surfactant, decreases the pulmonary surface. Further studies are needed to identify the exact role of GHR in hyaline membrane disease. It would be interesting to clarify if GHR has an anti-inflammatory or pro-inflam- matory effect in this lung pathological condition. In this relation, Li et al., reported that GHR protects alveolar macrophages against apoptosis in vivo and in vitro by its receptor GHSR-1a, expressed by the macrophages, which helps decrease the severity of sepsis-induced acute respiratory distress syndrome (ARDS) [32]. Their results indicate that GHR is a reliable candidate for use in a novel therapy strategy in sepsis-induced ARDS. Waseem et al., showed that GHR, by its receptors localized in neutrophils, lymphocytes, and macrophages, can potentially influence the immune system and that the administration of exogenous GHR might improve pathological inflammatory conditions [33]. There is also evidence that GHR protects against bleomycin-induced ARDS by reducing the apoptosis of alveolar epithelial cells and influence lung inflammation [34]. Taking into account the mentioned studies, we may hypothesize a similar role of GHR in RDS in newborns.

When lungs with RDS only were compared to the lungs with RDS plus pneumonia, we detected that in the alveolar septa of lungs with RDS only, the number of MCsGHR+ is lower than in patients who suffered both RDS and pneumonia. We also estimated that in patients with RDS+ pneumonia, the amount of MCsGHR+ and MCsTry+ in bronchioles was higher than in RDS only but without statistical significance. These results can be explained by the findings of $\mathrm{Xu}$ et al., who reported that, MCs are critical for the containment of and recovery from airway mycoplasma infection in mice [35]. According to these authors, although mast cells have phagocytic potential, it is unlikely that they can attract the immune cells on the luminal side of the airway in order to kill microbial cells, taking into account that most mast cells are localized under the epithelium and that their numbers within the epithelium or lumen are too small in normal conditions compared with resident macrophages. M. pneumoniae can be associated with significantly greater numbers of mast cells in a rodent mast cell line [36]. It is suggested that the pathogen can induce activation of mast cells leading to release of serotonin and $\beta$-hexosaminidase. Li et al. showed that both systemic and tissue-associated expression of GHR and GHSR1a is regulated during states of acute and chronic inflammation [32].

According to us, another explanation of MCs role in RDS is based on their ability to produce angiogenic factors such as VEGF which is responsible for tissue remodelling especially after blood vessel injury occurred in this pathological condition [37].

Mast cell membrane receptors can be targeted with suitable pharmaceutical agents that may modulate their function which can be useful in the treatment of RDS. 


\section{CONCLUSION}

The present study showed that MCs are another source of ghrelin different from neuroendocrine cells in human lung. The elevated number of tryptase positive mast cells in alveoli and bronchioles suggest that mast cells may play a role in the pathogenesis of RDS as well as are related to containment of and recovery of this pathological condition. However, increased amount of ghrelin positive mast cells and the role of ghrelin in this lung disease are needed to be clarified. This multifunctional hormone enhances mast cells significance and may help to elucidate the mechanism by which these cells maintain the lung homeostasis as well as to develop more reliable methods for diagnosing, prevention and treatment of RDS and other respiratory diseases.

\section{Disclosure summary: The authors have nothing to disclose.}

\section{REFERENCES}

1. Grappone L, Messina F. Hyaline membrane disease or respiratory distress syndrome? A new approach for an old disease Lidia. Journal of Pediatric and Neonatal Individualized Medicine 2014; 3: e030263.

2. Bitterman PB. Pathogenesis of fibrosis in acute lung injury. Am J Med 1992; 92: 39-43.

3. Locci G, Fanos V, Gerosa C, Faa G. Hyaline membrane disease (HMD): the role of the perinatal pathologist. Review. Journal of Pediatric and Neonatal Individualized Medicine 2014; 3: e030255.

4. Jo HS. Genetic risk factors associated with respiratory distress syndrome. Korean J Pediatr 2014; 57: 157-63.

5. Kojima M, Kangawa K. Ghrelin: structure and function. Physiol Rev 2005; 85: 495-522.

6. Wang G, Lee HM, Englander E, et al. Ghrelin-not just another stomach hormone. Regul Pept 2002; 105: 75-81.

7. Hattori N. Expression, regulation and biological actions of growth hormone $(\mathrm{GH})$ and Ghrelin in the immune system. Growth Horm IGF Res 2009; 19: 187-97.

8. Stefanov I, Ananiev J, Ivanova K, et al. Distribution of Ghrelinpositive mast cells in rat stomach. Biotechnology \& Biotechnological Equipment 2017; 31: 774-81.

9. Lambrecht BN, Hammad $\mathrm{H}$. The airway epithelium in asthma. Nat Med 2012; 18: 684-92.

10. Neil WS. Recent advances in our understanding of human host responses to tuberculosis. Respir Res 2001; 2: 157-163.

11. Yoshida T, Tuder RM. Pathobiology of cigarette smoke-induced chronic obstructive pulmonary disease. Physiol Rev 2007; 87: 1047-82

12. Sinnamon MJ, Carter KJ, Sims LP, et al. A protective role of mast cells in intestinal tumorigenesis. Carcinogenesis 2008; 29: 880-86.

13. Galli SJ, Tsai M. IgE and mast cells in allergic disease. Nat Med 2012; 18: 693-704.

14. 14. Boesiger J, Tsai M, Maurer M, et al. Mast cells can secrete vascular permeability factor/vascular endothelial cell growth factor and exhibit enhanced release after immunoglobulin Edependent upregulation of Fc epsilon receptor I expression. J Exp Med. 1998; 188: 1135-45.

15. Horsmanheimo L, Harvima IT, Jarvikallio A, et al. Mast cells are one major source of interleukin-4 in atopic dermatitis. $\mathrm{Br} \mathrm{J}$ Dermatol 1994; 131: 348-53.

16. Gibbs BF, Arm JP, Gibson K, et al. Human lung mast cells release small amounts of interleukin- 4 and tumour necrosis factor-alpha in response to stimulation by antilgE and stem cell factor. Eur J Pharmacol 1997; 327: 73-78.

17. Leon A, Buriani A, Dal Toso R, et al. Mast cells synthesize, store, and release nerve growth factor. Proc Natl Acad Sci USA 1994; 91: 3739-43.

18. Orinska Z, Maurer M, Mirghomizadeh F, et al. IL-15 constrains mast cell-dependent antibacterial defenses by suppressing chymase activities. Nat Med 2007; 13: 927-34.

19. Church MK, Levi-Schaffer F. The human mast cell. J Allergy Clin Immunol 1997; 99: 155-60.

20. Abraham SN, Thankavel K, Malaviya R. Mast cells as modulators of host defense in the lung. Front Biosci 1997; 2: 78-87.

21. Lan RS, Stewart GA, Henry PJ. Role of protease-activated receptors in airway function: a target for therapeutic intervention? Pharmacol Ther 2002; 95: 239-57.

22. Berger P, Girodet PO, Begueret H, et al. Tryptase-stimulated human airway smooth muscle cells induce cytokine synthesis and mast cell chemotaxis. FASEBJ 2003; 17: 2139-41.

23. Smith TJ, Hougland MW, Johnson DA. Human lung tryptase. Purification and characterization. J biol Chem 1984; 259: 11046-51.

24. McDonald DM, Schoeb TR, Lindsey JR. Mycoplasma infections cause long-lasting potentiation of neurogenic inflammation in the respiratory tract of the rat. J Clin Invest 1991; 87: 787-99.

25. Shimada K, Chen S, Dempsey PW, et al. The NOD/RIP2 Pathway Is Essential for Host Defenses Against Chlamydophila pneumoniae Lung Infection. PLoS Pathog 2009; 5: e1000379.

26. Rodriguez N, Fend F, Jennen L, et al. Polymorphonuclear neutrophils improve replication of Chlamydia pneumoniae in vivo upon MyD88-dependent attraction. J. Immunol 2005; 174: 4836-44.

27. Crother TR, Ma J, Jupelli M, et al. Plasmacytoid dendritic cells play a role for effective innate immune responses during Chlamydia pneumoniae infection in mice. PLoS One 2012; 7: e48655.

28. Pesci A, Majori M, Piccoli ML, et al. Mast cells in bronchiolitis obliterans organizing pneumonia. Mast cell hyperplasia and evidence for extracellular release of tryptase. Chest 1996; 110: 383-91.

29. Fisher MM, Baldo BA. Mast cell tryptase in anaesthetic anaphylactoid reactions. British Journal of Anaesthesia 1998; 80: 26-9.

30. Bradding $P$, Walls AF, Holgate ST. The role of the mast cell in the pathophysiology of asthma. J Allergy Clin Immunol 2006; 117: 1277-84.

31. Volante M, Fulcheri E, Allia E, et al. Ghrelin expression in fetal, infant, and adult human lung. J Histochem Cytochem 2002; 50: 1013-21.

32. Li B, Zeng M, He W, et al. Ghrelin protects alveolar macrophages against lipopolysaccharide-induced apoptosis through growth hormone secretagogue receptor 1a-dependent c-Jun $\mathrm{N}$-terminal kinase and Wnt/ $\beta$-catenin signaling and suppresses lung inflammation. Endocrinology 2015; 156: 203-17.

33. Waseem T, Duxbury M, Ito $\mathrm{H}$, et al. Exogenous Ghrelin modulates release of pro-inflammatory and anti-inflammatorycytokinesinLPS-stimulatedmacrophagesthroughdistinct signaling pathways. Surgery 2008; 143: 334-342.

34. Imazu Y, Yanagi S, Miyoshi K, et al. Ghrelin ameliorates bleomycin-induced acute lung injury by protecting alveolar epithelial cells and suppressing lung inflammation. Eur J Pharmacol 2011; 672: 153-8.

35. Xu X, Zhang D, Lyubynska N, et al. Mast cells protect mice from Mycoplasma pneumonia. Am J Respir Crit Care Med 2006; 173: 219-25.

36. Hoek KL, Cassell GH, Duffy LB, et al. Mycoplasma pneumoniaeinduced activation and cytokine production in rodent mast cells. J Allergy Clin Immunol 2002; 109: 470-76.

37. Aroni K, Voudouris S, loannidis E, et al. Increased angiogenesis and mast cells in the centre compared to the periphery of vitiligo lesions. Arch Dermatol Res 2010; 302: 601-07. 